NBER WORKING PAPER SERIES

\title{
TOWARD AN UNDERSTANDING OF THE RELATIVE STRENGTHS OF POSITIVE AND NEGATIVE RECIPROCITY
}

\author{
Omar Al-Ubaydli \\ Uri Gneezy \\ Min Sok Lee \\ John A. List \\ Working Paper 16547 \\ http://www.nber.org/papers/w16547 \\ NATIONAL BUREAU OF ECONOMIC RESEARCH \\ 1050 Massachusetts Avenue \\ Cambridge, MA 02138 \\ November 2010
}

We wish to thank Ali Hortacsu and an anonymous referee for helpful comments, Jonathan Alevy for programming help and Nicholas Curott for excellent research assistance. The views expressed herein are those of the authors and do not necessarily reflect the views of the National Bureau of Economic Research.

NBER working papers are circulated for discussion and comment purposes. They have not been peerreviewed or been subject to the review by the NBER Board of Directors that accompanies official NBER publications.

(C) 2010 by Omar Al-Ubaydli, Uri Gneezy, Min Sok Lee, and John A. List. All rights reserved. Short sections of text, not to exceed two paragraphs, may be quoted without explicit permission provided that full credit, including (C) notice, is given to the source. 
Toward an understanding of the relative strengths of positive and negative reciprocity Omar Al-Ubaydli, Uri Gneezy, Min Sok Lee, and John A. List

NBER Working Paper No. 16547

November 2010

JEL No. C9,C91

\begin{abstract}
A stylized fact is that agents respond more acutely to negative than positive stimuli. Such findings have generated insights on mechanism-design, have been featured prominently in policymaking, and more generally have led to discussions of whether preferences are defined over consumption levels or changes in consumption. This study reconsiders this stylized fact. In doing so, it provides insights into an important domain wherein positive stimuli induce a greater response than negative stimuli: a principal-agent game with reputational considerations and with the agent on the market's short end. This common setting represents an important feature of labor markets with involuntary unemployment.

$\begin{array}{ll}\begin{array}{l}\text { Omar Al-Ubaydli } \\ \begin{array}{l}\text { Department of Economics and Mercatus Center } \\ \text { George Mason University }\end{array} \\ \text { The Kenneth and Anne Griffin Foundation } \\ \text { Min.Lee@ @riffin-foundation.com }\end{array} \\ \begin{array}{ll}\text { John A. List } \\ \text { Uri Gneezy }\end{array} \\ \begin{array}{l}\text { Department of Economics } \\ \text { Rady School of Management }\end{array} & \text { University of Chicago } \\ \text { University of California - San Diego } & \text { 1126 East 59th } \\ \text { Otterson Hall, Room 4S136 } & \text { Chicago, IL 60637 } \\ \text { 9500 Gilman Drive \#0553 } & \text { and NBER } \\ \text { La Jolla, CA 92093-0553 } & \text { jlist@ uchicago.edu } \\ \text { ugneezy@ ucsd.edu } & \end{array}$
\end{abstract}




\section{Introduction}

The principle of retaliation is as old as mankind. As far back as the Hammarabian code some 3000 years ago, retaliation of some form has served to organize behavior in both market and non-market situations. Perhaps illustrating the importance of revenge most succinctly is the Biblical injunction of Exodus 21:2325: "Life for life, eye for eye, tooth for tooth... bruise for bruise." For their part, scholars have explored the importance of negative actions alongside their seemingly more benign cousins, positive actions.

One of the key insights that can be taken from the decades of research within the social sciences is that reciprocity in general is important, and that negative actions toward an individual induce a greater behavioral response than comparable positive actions. ${ }^{2}$ This stylized fact is perhaps best illustrated in the words of Baumeister et al. (2001), who provide a broad survey of several areas of study examining positive and negative reciprocity, and conclude that (p. 354-55, italics added): "The breadth and convergence of evidence, however, across different areas were striking, which forms the most important evidence. In no area were we able to find a consistent reversal, such that one could draw a firm conclusion that good is stronger than bad. This failure to find any substantial contrary patterns occurred despite our own wishes and efforts... Hence, we must conclude that bad is stronger than good at a pervasive, general level."

Within economics, such results have served as the classic example of loss aversion - that people are more sensitive to negative realizations than to positive realizations of uncertainty (Tversky and Kahneman (1991)) - have played an important role in policymaking (see List (2003)), and have informed mechanism design. In terms of the latter, the principal is confronted with an interesting decision problem if framing of the incentive scheme matters to agent behavior or the number of instruments available to the principal is constrained. In this manner, choosing between carrots and sticks, for example, plays an important role in the outcome (see Andreoni et al. (2003)). More generally, scholars have frequently remarked that this feature of human preferences represents one of the most robust general behavioral patterns in the social sciences (see the citations in Baumeister et al. (2001)).

In this study, we explore a general setting wherein economic theory provides predictions that positive reciprocity should be stronger than negative reciprocity. The two key features are that the agent is on the short end of a market that includes reputational considerations and that being out of the market provides less utility than being a participant. Under this design, a worker that respects her initial affective reaction and punishes the employer will find herself unemployed. Alternatively, a worker who

\footnotetext{
${ }^{2}$ A particularly simple exposition involves no more than asking subjects to list emotions within a time limit (Van Goozen and Frijda (1993)). The number of negative emotions listed almost always exceeds positive ones. Along similar lines, Oehman et al. (2001) found that people identified threatening faces more quickly and accurately than happy faces. Such negative visual stimuli also induce larger amplitude brain responses than positive ones (Ito et al. (1998)). Generally, the negative domain commands affect and cognition more than the positive. In their survey, Baumeister et al. (2001) somewhat playfully draw our attention to Fiedler's (1982) finding that nobody has ever written a successful novel about a happy marriage; there is something about negative events that seizes our attention. Similar to scholars in other social sciences, economists have found that negative events also call forth greater responses than their positive counterparts (see, e.g., Offerman (2002), Pereira et al. (2006) and Al-Ubaydli and Lee (2009)).
} 
is nice to the employer will be more likely to be employed in the next period. Since being employed dominates unemployment, we predict that the worker will restrain herself and will not follow the initial affective reaction. On the other hand, if the employer is nice, the worker will reciprocate strongly since in this situation not only is she employed, but also by a nice employer. In other words, we predict that in our setup positive reciprocity will be stronger than negative reciprocity.

To test our theory, we design a simple controlled laboratory experiment, which yields several insights. First, consonant with the literature, agents reciprocate. And, when the interactions are anonymous, negative reciprocity is slightly more important than positive reciprocity, but not significantly so. Also consonant with the literature is the fact that agents become emotionally charged when treated poorly. Yet, this emotional charge does not readily transfer to actions when realistic institutional features are in place. For example, when agents can form reputations, they respond much more acutely to positive than to negative stimuli, serving to reverse the previously documented findings on the relative strengths of negative and positive reciprocity. Second, the data suggest that the source of the behavioral differences observed is strategic, rather than a change in the social norm of reciprocation in a gift exchange setting. Finally, the results also raise a methodological contribution of some import. Although the literature has implicitly drawn a parallel between the strength of reciprocal behaviors and other phenomena such as the Heisenberg Uncertainty Principle, our analysis highlights a critical distinction: unlike in quantum mechanics, people responding more acutely to negative than positive stimuli is not a guaranteed behavioral phenomenon. Rather, its presence and magnitude depend heavily on institutional features that can only be understood with appropriate theoretical and empirical modeling.

The remainder of our study proceeds as follows. Section 2 contains the experimental design. Section 3 summarizes the experimental results. Section 4 concludes.

\section{Experimental design}

To provide insights into measuring the strength of positive and negative reciprocity, we designed a simple two treatment experiment to test three conjectures. First, in one-shot environments, negative reciprocity is stronger than positive reciprocity. Second, in a repeated environment with a threat of exclusion, positive reciprocity is stronger than negative reciprocity. Finally, any observed difference in behavior across the two environments is not driven by affective reactions. In other words, when we condition on affective reactions, behavioral differences should remain.

The game played by subjects is a discrete version of the trust game (Berg et al. (1995)) where the agent (worker) has the opportunity to punish as well as reward. It is also repeated with an opportunity for principals (managers) to choose among the workers in each period. We begin by describing the simple stage game. 


\section{Stage game}

There are two players: a manager and a worker who play the stage game in Figure 1. In each column vector of Figure 1, the top (bottom) number is the manager's (worker's) payoff. The manager has three actions: kind, neutral and unkind. After the manager makes her choice, the worker observes the manager's choice and has three available actions: reward, neutral and punish. ${ }^{3}$ Similar to Offerman (2002), the payoff consequences of actions are separable across the choices of each player. If the manager plays neutral, then both players' payoffs are unchanged. If the manager plays kind, then she loses 10 points and the worker gains 15 points. If the manager plays unkind, then she gains 15 points and the worker loses 15 points. Note that kind and unkind have symmetric effects on the worker's payoff.

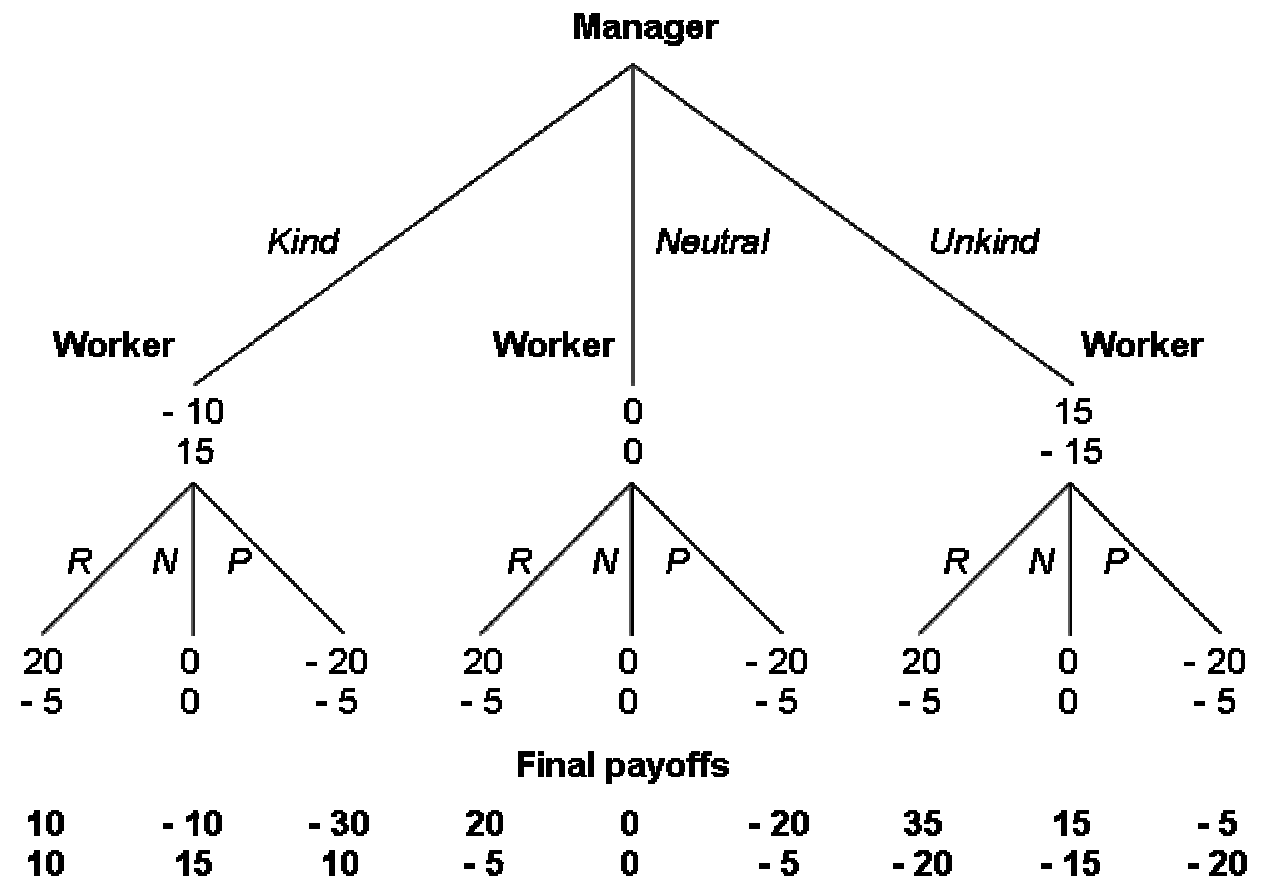

Figure 1: Stage game

In each column vector, top number is manager payoff, bottom number is worker payoff. Final payoffs are obtained by summing the each of the two vectors implied by the strategy. $R$ denotes reward, $N$ denotes neutral and $P$ denotes punishment.

At all nodes, if the worker plays neutral, both players' payoffs are unchanged. If the worker plays reward, she loses 5 points and the manager gains 20 points. If the worker plays punish, she loses 5 points and the manager loses 20 points. Thus reward and punish are equally costly to the worker and have symmetric effects on the manager's payoff (as in Offerman (2002)). Similar to most versions of the trust game, the unique subgame perfect equilibrium (assuming selfish preferences) is for the worker to

\footnotetext{
${ }^{3}$ In the experiment, roles and strategies were given neutral names. See below for more details.
} 
play neutral at all nodes and the manager to therefore play unkind. The unique symmetric efficient outcome is (kind, reward if kind).

\section{$\underline{\text { 2. Experimental game and treatments }}$}

The full game has 8 players: 3 managers and 5 workers. It is repeated for an uncertain number of periods. Throughout all versions of the game, the 3 managers have fixed IDs. In the key "reputation" treatment, workers also have fixed IDs, which are carried from period to period. In the one-shot, baseline treatment, the same fixed selection of IDs is randomly reassigned to each of the workers every round. For example, worker 3 might be a different person in round 1 to worker 3 in round 2 . These features are common knowledge.

Play proceeds in the following manner.

1. Nature decides the order in which each manager gets to select the worker that will be her partner (uniform distribution).

2. The first manager selects her partner; the next manager selects from the remaining workers, and so on.

- The 2 workers who are unselected for the round lose 25 points. This is 10 points less than the worst guaranteed payoff when selected. In other words, being employed by a nasty manager is better than being unemployed.

3. Each manager chooses between kind, neutral and unkind.

4. Each worker finds out which manager selected them and what choice the manager made. The workers who were not selected are informed of these choices.

5. Each paired worker chooses between reward, neutral and punish.

6. All players see their own payoffs for the round. They also see the choices made by all managerworker pairs in that round and they see the history of choices by all pairs.

- Recall that worker IDs are only fixed in the reputation treatment. In the one-shot treatment, it is common knowledge that, say, worker 5's choice in round 3 may not have been made by the same player as worker 5 's choice in round 4 .

The game is repeated for a total of 11 rounds, though at the start of the experiment to prevent endgame effects subjects are told only that the experiment will continue for a "number of rounds." Moreover, the decision-making stages of all sessions end at least 25 minutes in advance of the 90 minutes for which subjects sign up (it takes about 10 minutes to calculate earnings and pay subjects).

The reputation treatment is designed to capture a situation where reputational concerns - allied with an exclusion threat - will make positive reciprocity dominate negative reciprocity. The one-shot treatment is designed as a control where the elimination of reputational concerns and strategic exclusion will move the balance back towards the stylized fact, i.e., dominance of negative reciprocity.

To explore affective reactions, in some sessions we asked each paired worker to declare privately how they feel about their manager's choice: very unhappy, somewhat unhappy, neutral, somewhat happy, 
very happy. ${ }^{4}$ This permits an exploration of whether affective reactions have a greater influence on reward/punishment decisions in the one-shot treatment. Since worker behavior was not different across the sessions where this information was elicited, we pool the data below.

\section{Procedure}

In total, we ran 9 sessions at George Mason University during spring 2009. Subjects were recruited from a database of students who had declared an interest in participating in economics experiments. Each session had 11 periods and 16 subjects divided into two groups of 8 ( 3 managers, 5 workers). Roles were assigned randomly and all interactions were anonymous. Subjects' roles were fixed and they interacted exclusively with members of their own group. The total number of worker observations collected is therefore $591 .^{5}$

The experiment was computer-based and used z-Tree (Fischbacher (2007)). Instructions were on-screen, though a hard copy was given to each subject. All subjects were in the same room and within earshot and eyeshot of each other. This is potentially important because the monitor read the instructions aloud to ensure common knowledge. The game was presented in a neutral frame, i.e., managers chose between left, middle and right, and workers chose between add 20, nothing and subtract 20. Managers were called Reds and workers were called Blues. After completing the experiment, subjects were paid privately. ${ }^{6}$

\section{Empirical results}

Table 1 presents a summary of the experimental results. In this summary, to provide a first glimpse of behavioral patterns we have ignored data dependencies and pooled individual play over all 11 periods of the game. As we discuss each result below, we supplement these raw data patterns with conditional analysis. We begin with a first result.

Result 1: In both treatments, workers reciprocate manager choices: they frequently reward kind and punish unkind.

Evidence to support this result can be seen in Table 1, where it is shown that workers reward kind actions in $51 \%$ of the cases in the one-shot treatments and in $84 \%$ of the cases in the reputation

\footnotetext{
${ }^{4}$ The only part of the game that was not common knowledge was asking about emotions; it was not read out at the start (see procedure below) and only appeared on the workers' screens. This was done to minimize priming of the subjects to think in terms of affective reactions. As noted, for robustness we also ran sessions where we did not ask the workers to declare their emotions. None of the results were affected.

${ }^{5}$ The treatment breakdown was 396 from one-shot and 195 from reputation. We lost the data from the last period of one group in one session (3 observations).

${ }^{6}$ To compensate for the fact that some subjects had a negative payoff, there was a large show-up fee, though this was only announced after the start of the experiment, i.e., subject recruitment utilized the usual GMU show-up fee. Average earnings were approximately $\$ 18$.
} 
treatment. Likewise, they punish unkind actions in 53\% of the instances they occur in the one-shot treatment and in $34 \%$ of the instances they occur in the reputation treatments. ${ }^{7}$ These figures are both significantly higher than the propensities to react positively or negatively to other manager actions (i.e., the percentage of reward play in response to a manager choosing neutral), and are all significantly different from zero using conventional parametric statistical tests at the $p<1 \%$ level. Similar results are found when using nonparametric tests that have a null hypothesis of no treatment effect, or that the two samples are derived from identical populations.

\begin{tabular}{|l|c|c|}
\hline Number of sessions & One-shot & Reputation \\
\hline Relative frequency of Kind & 6 & 3 \\
\hline Relative frequnecy of Unkind & $36 \%$ & $53 \%$ \\
\hline Relative frequency of rewarding Kind & $51 \%$ & $84 \%$ \\
\hline $\begin{array}{l}\text { Relative frequency of punishing Un- } \\
\text { kind }\end{array}$ & $53 \%$ & $34 \%$ \\
\hline $\begin{array}{l}\text { Average emotion given Kind } \\
\text { Average emotion given Unkind }\end{array}$ & 1.7 & 1.8 \\
\hline $\begin{array}{l}\text { Relative frequency of reselection if } \\
\text { rewarded Kind last period }\end{array}$ & -1.6 & -1.4 \\
\hline $\begin{array}{l}\text { Relative frequency of reselection if } \\
\text { did not reward Kind last period }\end{array}$ & $64 \%$ & $91 \%$ \\
\hline $\begin{array}{l}\text { Relative frequency of reselection if } \\
\text { punished Unkind last period }\end{array}$ & $56 \%$ & $54 \%$ \\
\hline $\begin{array}{l}\text { Relative frequency of reselection if } \\
\text { did not punish Unkind last period }\end{array}$ & $61 \%$ & $61 \%$ \\
\hline
\end{tabular}

Table 1: Summary statistics by treatment

Emotions are scaled as follows: $-2=$ very unhappy, $-1=$ somewhat unhappy, $0=$ neutral, $+1=$ somewhat happy, $+2=$ very happy.

\footnotetext{
${ }^{7}$ All of our inference (for all the results) excludes the data corresponding to managers selecting neutral since it is not relevant to comparisons of positive and negative reciprocity. For the interested reader, here are a few features of the excluded data. Mangers play neutral $8 \%$ of the time. In both treatments, worker emotions in response to neutral are insignificantly different from 0 (on a scale of -2 to +2 ) using a t-test $(p>30 \%$ ).
} 
These unconditional tests treat observations as IID. As a robustness check, we estimate conditional parametric models that include period and group fixed-effects as well as individual clusters. ${ }^{8}$ The conditional results echo the unconditional results. For parsimony, we relegate them to the appendix.

This result is not surprising, as scores of studies have found reciprocal behavior - from student subjects to CEOs (see, e.g., Offerman (2002), Andreoni et al. (2003), Fehr and List (2004)). Examining the data at a slightly deeper level, we observe another result that is in line with the literature.

Result 2: In the one-shot treatment, negative reciprocity is slightly stronger than positive reciprocity, though the difference is statistically insignificant.

Evidence to support this result can be seen in Tables 1 and 2. In Table 1, for example, we find that in the one-shot treatment, workers reward kind actions in $51 \%$ of cases, while they punish unkind at the slightly higher rate of $53 \%$ of cases, though this difference is insignificant using Mann-Whitney and ttests.

In Table 2, we demonstrate this result using conditional tests with individual clusters. Let the outcome variable be a dummy variable taking the value ' 1 ' if a worker reciprocates, i.e., responds to kind with reward or responds to unkind with punish. We denote this variable reciprocate. Let the control group be the observations where the manager plays unkind and let the treatment group be the observations where the manager plays kind (both in the one-shot sessions).

The estimated coefficient on 'positive reciprocity' should be read as how much more likely a worker is to reward kind than she is to punish unkind. Using both a linear probability model (model 1) and a probit model (model 2), punishing unkind is $9 \%$ more likely than rewarding kind, though this difference is again insignificant $(p=31 \%)$. This is directionally consistent with the existing literature's finding that in one-shot environments, the negative reciprocity is stronger than positive reciprocity (Offerman (2002), Al-Ubaydli and Lee (2009)). ${ }^{9}$ Our next result departs from the existing literature.

Result 3: In the reputation treatment, positive reciprocity is stronger than negative reciprocity.

Evidence to support this result can be seen in Tables 1 and 2. Looking at Table 1, we see that in the reputation treatment, unconditionally, a worker is $50 \%$ more likely to reward kind than she is to punish unkind. Both Mann-Whitney and t-tests are significant at conventional levels ( $p<1 \%)$.

The conditional results with individual clusters are contained in Table 2. Again, the estimated coefficient on 'positive reciprocity' should be interpreted as how much more likely a worker is to reward kind than she is to punish unkind. Using both a linear probability model (model 3) and a probit model (model 4), we find that the estimated coefficient is over $50 \%$ and statistically significant $(p<1 \%)$.

\footnotetext{
${ }^{8}$ In all our parametric models, we use two clusters per worker: one for the worker's decision at the kind node and one at the unkind node. Using a common cluster instead affects none of our results (in fact using a common cluster shrinks the standard errors - as one would expect - though not by enough to alter any result).

${ }^{9}$ Essentially all our results are robust to using only data from periods 6-to-11, reinforcing the design's attempts at avoiding any 'end-of-session' effects. The only slight exception is result 4 . See below.
} 


\begin{tabular}{|c|c|c|c|c|c|c|c|c|c|c|c|}
\hline & Model 1 & Model 2 & Model 3 & Model 4 & Model 5 & Model 6 & Model 7 & Model 8 & Model 9 & Model 10 & Model 11 \\
\hline Estimation method & Regression & Probit & Regression & Probit & Regression & Probit & Regression & Regression & Probit & Regression & Probit \\
\hline Dependent variable & Reciprocate & Reciprocate & Reciprocate & Reciprocate & Reciprocate & Reciprocate & Emotions & Reselect & Reselect & Reselect & Reselect \\
\hline Positive reciprocity & -0.09 & -0.09 & $0.51^{* * *}$ & $0.54^{* * *}$ & -0.08 & -0.08 & $3.26^{* * *}$ & - & - & - & - \\
\hline Standard error & $(0.09)$ & $(0.09)$ & $(0.09)$ & $(0.08)$ & $(0.09)$ & $(0.09)$ & $(0.22)$ & - & - & - & - \\
\hline Reputation session & - & - & - & - & -0.21 & -0.22 & 0.09 & - & - & - & - \\
\hline Standard error & - & - & - & - & $(0.20)$ & $(0.21)$ & $(0.30)$ & - & - & - & - \\
\hline Positive reciprocity $x$ Reputotion session & - & - & - & - & $0.56^{* * *}$ & $0.47^{* * * *}$ & -0.09 & - & - & - & - \\
\hline Standard error & - & - & - & - & $(0.13)$ & $(0.07)$ & $(0.29)$ & - & - & - & - \\
\hline Rewarded kind last period & - & - & - & - & - & - & - & $0.36^{* * k}$ & $0.40^{* * * *}$ & - & - \\
\hline Standard error & - & - & - & - & - & - & - & $(0.13)$ & $(0.21)$ & - & - \\
\hline Punished unkind last period & - & - & - & - & - & - & - & - & - & $-0.25^{* *}$ & $-0.30^{* * * *}$ \\
\hline Standard error & - & - & - & - & - & - & - & - & - & $(0.11)$ & $(0.10)$ \\
\hline Dota included & One-shot & One-shot & Reputation & Reputation & Pooled & Pooled & Pooled & Reputation & Reputation & Reputation & Reputation \\
\hline$R^{2} /$ Pseudio $R^{2}$ & 0.11 & 0.09 & 0.32 & 0.26 & 0.16 & 0.13 & 0.80 & 0.26 & 0.27 & 0.25 & 0.20 \\
\hline Observations & 360 & 360 & 182 & 182 & 542 & 542 & 233 & 77 & 77 & 87 & 87 \\
\hline
\end{tabular}

\section{Table 2: Conditional results}

The dummy variable 'Reciprocate' takes the value 1 when the worker reciprocates kind with reward or unkind with punish. Emotions are scaled as follows: $-2=$ very unhappy, $-1=$ somewhat unhappy, $0=$ neutral, $+1=$ somewhat happy, $+2=$ very happy. The dummy variable 'Reselect' takes the value 1 when a worker is reselected by (any) manager in that period given selection in the previous period. 'Positive reciprocity' is a dummy variable that takes the value 0 when the manager plays unkind and 1 when the manager plays kind. 'Reputation session' is a dummy that takes the value 1 in reputation sessions. In probits, the reported figure is the estimated marginal effect. All models contain clusters at the individual level. Group effects correspond to the fact that each session has two groups. All models exclude the 49 observations corresponding to the manager playing neutral. Estimated period/group fixed effects are omitted. Asterices denote statistical significance $(*=10 \%, * *=5 \%, * *=1 \%)$. 
This result stands in stark contrast to results in the literature, and questions the generality of bad being stronger than good. And, it provides the empirical reversal that has alluded researchers who have been unable to locate such an example (Baumeister et al. (2001)). Results 4-to-6 attempt to shed light on the underpinnings for this reversal.

Result 4: The difference in reciprocity between one-shot and reputation sessions is driven primarily by a large increase in positive reciprocity when going from one-shot to reputation sessions.

Empirical evidence to support this result can be seen in Table 1, where positive reciprocity increases by $34 \%$ when moving from the one-shot to the reputation treatment, whereas punishment falls by $19 \%$. Testing this formally requires a conditional parametric specification. In models 5 and 6 in Table 2, we pool the data from our one-shot and reputation treatments and include a reputation by session dummy variable and an interaction term between positive reciprocity and the reputation by session dummy.

The reputation by session dummy coefficient informs tells us how much more likely workers are to punish unkind in the reputation treatment than in the one-shot treatment. The point estimates, of roughly $20 \%$, suggest that workers are substantially less likely to punish in the reputation treatment, though this is not statistically significant at conventional levels. Thus negative reciprocity is at most slightly smaller in the reputation treatment than in the one-shot treatment.

Given this result, the large (greater than $46 \%$ ) and significant $(p<1 \%$ ) coefficient of the interaction of the reputation and positive reciprocity is primarily the result of a substantial increase in positive reciprocity when going from one-shot to reputation. ${ }^{10}$ Our next result concerns the underpinnings of the reversal.

Result 5: The difference in the balance of positive and negative reciprocity across one-shot and reputation sessions is not the result of differences in the affective reactions to kind and unkind across one-shot and reputation sessions.

Evidence to support this result can be seen in Tables 1 and 2. Recall that the scale for worker's declared emotion after seeing the manager's choice is: $-2=$ very unhappy, $-1=$ somewhat unhappy, $0=$ neutral, $+1=$ somewhat happy,$+2=$ very happy.

In Table 1, the mean emotion in response to kind is +1.7 in the one-shot treatment and +1.8 in the reputation treatment. This difference is insignificant using three unconditional tests (Mann-Whitney: $p=$ $19 \%$, t-test: $p=30 \%$; Kolmogorov-Smirnov: $p=96 \%$ ). The mean emotion in response to unkind is -1.6 in the one-shot treatment and -1.4 in the reputation treatment. This difference is marginally significant or insignificant depending on the unconditional test employed (Mann-Whitney: $p=10 \%$, t-test: $p=12 \%$; Kolmogorov-Smirnov: $p=66 \%$ ).

In model 7 in Table 2, we estimate a regression model of emotions (with individual clusters) on three explanatory variables: 'positive reciprocity,' which takes the value 0 when the manager has played

\footnotetext{
${ }^{10}$ When we only use data from periods 6-to-11, in addition to the large increase in reward (73\%), there is a large (but still smaller) decrease in punishment (52\%). This does not affect the paper's main argument.
} 
unkind and 1 when the manager has played kind; a reputation session dummy; and an interaction between these two variables. In this model, the estimated coefficients on the reputation sessions dummy and on the interaction between positive reciprocity and reputation sessions dummies are statistically insignificant. In other words: worker emotive responses to manager choices do not depend upon being in one-shot vs. reputation treatments. Thus, the marginal significance levels obtained in a couple of the unconditional tests are the result of erroneously treating the observations as IID. ${ }^{11}$

Perhaps the most compelling evidence that the results are not driven by differences in affective reactions is that in the one-shot sessions, when the worker was very unhappy at the manager playing unkind, she rewarded the manager $3 \%$ of the time. The corresponding Figure for the reputation sessions was $17 \%$ ( $n=103, p<5 \%$ using a t-test and a MW-test). Clearly several workers were willing to reciprocate due to the threat of strategic exclusion in the reputation sessions.

This suggests that the difference in the balance of positive and negative reciprocity across one-shot and reputation sessions is not the result of differences in the affective reactions, leading to our next result.

Result 6: The difference in the balance of positive and negative reciprocity across one-shot and reputation sessions is the result of strategic differences in the environment, specifically the threat of systematic exclusion.

Evidence to support this result can be seen in Tables 1 and 2. The dimensionality of the history space is too large for sophisticated structural modeling (the data demands are not met either), however result 6 can still be derived from a more modest structural approach. The basic hypothesis is that in the reputation sessions, managers seek workers who have rewarded kind or who did not punish unkind.

In Table 1, for example, we see that in the reputation treatment, if a worker rewards a play of kind, then this increases her chances of being reselected by a manager in the subsequent round by $43 \%$ compared to not rewarding (significant at $p<3 \%$ using Mann-Whitney and t-tests). Similarly, if a worker punishes a play if unkind, then this decreases her chances of being reselected by a manger in the subsequent round by $24 \%$ compared to not punishing (significant at $p<3 \%$ using Mann-Whitney and $t$ tests). In the one-shot treatments, statistically speaking, reselection chances are unaffected by past play. This is comforting since the worker IDs were scrambled every round and so the managers could not perform any systematic exclusion.

Table 2 reveals that conditional models with individual clusters yield consistent results. Models 8 and 9 confirm that rewarding kind increases a worker's probability of being selected in the next round by over $35 \%$. On the negative reciprocity side, models 10 and 11 confirm that punishing unkind diminishes a worker's probability of being reselected by over $24 \%$. Again, re-estimating models 8-11 using data from the one-shot sessions (omitted for parsimony), we find that all coefficients are statistically insignificant (all have a p-value greater than $50 \%$ ) and have very small magnitudes (smaller than $5 \%$ ).

\footnotetext{
${ }^{11}$ A minor result that we omit for parsimony is that in both treatments, emotions predict reward and punishment in the expected way, i.e., subjects who report more positive emotions are more likely to reward and less likely to punish.
} 
While it is clear that managers account for worker actions in their partnerships choices, another manner in which managers' behavior potentially changes is due to treatment itself. Our data reveal that workers punish much less often but reward more frequently in the repeated game than in the one shot game. An interesting query is whether managers use this information effectively in their choices.

Given the unconditional relative frequencies of reward and punishment behavior in the one-shot treatment, and treating workers choices as IID draws, then for managers we have that unkind $>$ kind $>$ neutral, where $>$ denotes strict preference under expected-payoff maximization. This is loosely reflected in their choices (see Table 1): unkind (54\%), kind (36\%), neutral (9\%). The comparatively large incidence of kind is consonant with equity considerations.

In the reputation treatment, both the optimal and realized rank-ordering of manager choices is unchanged. While there is a slight increase (7\%) in kind at the expense of neutral and unkind, none of the changes are statistically significant using conditional or unconditional tests. To some extent, this is unsurprising since increased reward and decreased punishment renders kind and unkind simultaneously more lucrative in absolute terms. This exploration leads to our final result.

Result 7: Efficiency is substantially higher in the reputation treatment.

The total realized payoff in the one-shot treatment is $14 \%$ of the total potential payoff, while the corresponding Figure for the reputation treatment is $40 \%$. The reason why both are so low is because any deviation from kind and reward leads to a lower aggregate payoff, and such deviations are very frequent. Naturally, the efficiency improvement is driven by workers moving away from punishment towards reward.

\section{Conclusion}

As Arrow (1972, p. 357) put forth decades ago when he noted that "Virtually every commercial transaction has within itself an element of trust," most economic and non-economic transactions require a degree of trust. With the element of trust comes the necessary ingredient reciprocity. Scholars as far back as Aristotle (2004) appreciated the importance of negative reciprocity, as he extolled that revenge serves to discourage mistreatment. More recently, scientists have come to the firm conclusion that both negative and positive reciprocity are important, but that at a general level the relative strengths are not comparable: bad is stronger than good.

This paper revisits this stylized fact by infusing two realistic features - the agent is on the short end of a market that includes reputational considerations and that being out of the market provides less utility than being a participant - into a popular laboratory game. We argue that these additional considerations provide a setting that is representative of many common economic situations. This alteration permits us to examine the relative strengths of positive and negative reciprocity while simultaneously exploring the underpinnings for reciprocity. 
We find that the stylized fact in the literature is reversed in our setting: in repeated environments with a threat of systematic exclusion, good is stronger than bad. ${ }^{12}$ This holds because in our setting being employed by an exploitative manager dominates unemployment. Rational agents understand that this is the case and act accordingly. Importantly, this reversal is not the consequence of a change in affective reactions. People are as happy about kind behavior in the repeated environment as they are in the oneshot environment, and they are equally riled by unkind behavior across the two environments. Rather, the prominence of positive reciprocity in the repeated environment is driven by strategic concerns: those workers who are cooperative - either by reciprocating kind behavior or refraining from punishing unkind behavior - avoid unemployment by acting appropriately in the environment.

We view these results as important in several domains. First, they move us toward a deeper understanding of the relative strengths of positive and negative reciprocity. In this way, the received results highlight the importance of the economic and psychological features embedded in any economic environment. Second, in doing so they open new paths of inquiry. For instance, in public policymaking, the general discussion of whether preferences are defined over consumption levels or changes in consumption has moved policymakers to more carefully consider the differences between willingness to pay and willingness to accept in cost benefit analysis. Understanding the mechanisms that underlie these valuation divergences is invaluable. Also, the practitioner interested in mechanism design might regard the results of import when crafting incentive schemes to alter agent behavior.

\section{References}

Al-Ubaydli, O., and M. Lee (2009). "An experimental study of asymmetric reciprocity," Journal of Economic Behavior and Organization. Forthcoming.

Andreoni, J., W. Harbaugh and L. Vesterlund (2003). "The carrot or the stick: rewards, punishments, and cooperation," American Economic Review. 93, p893-902.

Aristotle (2004). Rhetoric (Book II), translated by W. Rhys Roberts. Dover Publications.

Arrow, K. (1972). "Gift and exchanges," Philosophy and Public Affairs. 1, p343-362.

Baumeister, R., E. Bratslavsky, C. Finkenauer and K. Vohs (2001). "Bad is stronger than good," Review of General Psychology. 5, p323-370.

Berg, J., J. Dickhaut and K. McCabe (1995). "Trust, reciprocity and social history," Games and Economic Behavior. 10, p122-142.

\footnotetext{
${ }^{12}$ A related study is Rand et al. (2009), which finds that in repeated a public goods game, reward is superior to punishment in eliciting cooperation. Also, see Kube et al. (2006) for a field comparison of positive and negative reciprocity in a one-shot setting.
} 
Fehr, E. and J. List (2004). "The hidden costs and returns of incentives - trust and trustworthiness among CEOs," Journal of European Economic Association. 2, p743-771.

Fiedler, L. (1982). Love and death in the American novel. Stein \& Day, New York.

Fischbacher, U. (2007). "z-Tree: Zurich toolbox for ready-made economic experiments," Experimental Economics. 10, p171-178.

Ito, T. , J. Cacioppo and P. Lang (1998). "Eliciting affecting using the International Affective Picture System: Bivariate evaluation and ambivalence," Personality and Social Psychology Bulletin. 24, p855879.

Kube, S., M. Marechal and C. Puppe (2006). "Putting reciprocity to work - positive versus negative responses in the field," Mimeo, University of Karlsruhe.

List, J. (2003). “Does market experience eliminate market anomalies?,” Quarterly Journal of Economics. $118, \mathrm{p} 41-71$.

Oehman, A., D. Lundqvist and F. Esteves (2001). "The face in the crowd revisited: A threat advantage with schematic stimuli," Journal of Personality and Social Psychology. 80, p381-396.

Offerman, T. (2002). "Hurting hurts more than helping helps," European Economic Review. 46, p14231437.

Pereira, P., N. Silva and J Silva (2006). "Positive and negative reciprocity in the labor market," Journal of Economic Behavior and Organization. 59, p406-422.

Rand, D., A. Dreber, T. Ellingsen, D. Fudenberg and M. Nowak (2009). "Positive interactions promote public cooperation," Science. 325, p1272-1275.

Tversky, A. and D. Kahneman (1991). "Loss aversion in riskless choice: a reference-dependent model," Quarterly Journal of Economics. 106, p1039-1061.

Van Goozen, S. and N. Frijda (1993). "Emotion words used in six European countries," European Journal of Social Psychology. 23, p89-95. 


\section{Appendix}

\section{Conditional results for result 1}

\begin{tabular}{|c|c|c|c|c|c|c|c|c|}
\hline & Model 1 & Model 2 & Model 3 & Model 4 & Model 5 & Model 6 & Model 7 & Model 8 \\
\hline Estimation method & Regression & Probit & Regression & Probit & Regression & Probit & Regression & Probit \\
\hline Dependent variable & Reward & Reward & Reward & Reward & Punish & Punish & Punish & Punish \\
\hline Dummy denoting manager playing Kind & $0.43^{* * *}$ & $0.55 * * *$ & $0.64 * * *$ & $0.81 * * *$ & -0.11 & $-0.24 * *$ & $-0.15^{*}$ & $-0.22^{* *}$ \\
\hline Standard error & $(0.09)$ & $(0.15)$ & $(0.14)$ & $(0.11)$ & $(0.09)$ & $(0.09)$ & $(0.09)$ & $(0.08)$ \\
\hline Dummy denoting manager playing Unkind & 0.04 & 0.07 & -0.03 & 0.03 & $0.39 * * *$ & $0.32 * * *$ & 0.13 & 0.08 \\
\hline Standard error & $(0.08)$ & $(0.12)$ & $(0.13)$ & $(0.23)$ & $(0.10)$ & $(0.09)$ & $(0.10)$ & $(0.07)$ \\
\hline Data included & One-shot & One-shot & Reputation & Reputation & One-shot & One-shot & Reputation & Reputation \\
\hline$R^{2} /$ Pseudo $R^{2}$ & 0.35 & 0.36 & 0.58 & 0.52 & 0.40 & 0.29 & 0.35 & 0.31 \\
\hline Observations & 396 & 396 & 195 & 195 & 396 & 396 & 195 & 195 \\
\hline
\end{tabular}

\section{Table A1: Conditional results for result 1}

The dummy variable 'Reward' takes the value 1 when the worker rewards. The dummy variable 'Punish' takes the value 1 when the worker punishes. In probits, the reported figure is the estimated marginal effect. All models contain clusters at the individual level. Group effects correspond to the fact that each session has two groups. Estimated period/group fixed effects are omitted. All models exclude the 49 observations corresponding to the manager playing neutral. Asterices denote statistical significance $(*=10 \%, * *=5 \%, * * *=1 \%)$. 


\section{Experimental instructions}

Welcome to our experiment in decision making.

If you read these instructions carefully and make good decisions, you may earn a considerable amount of money. At the end of the experiment, your earnings will be paid to you, privately and in cash.

At the beginning of the experiment, you will be randomly separated into groups of 8. You will only interact with your group members. Three of you will be randomly assigned the role of Red and five of you the role of Blue. So there are more Blues than Reds. [NEXT]

At the beginning of the experiment, both Reds and Blues get 200 points each as a show-up fee. We will convert the points you earned into dollars at the rate of 10 points $=\$ 1$. [NEXT]

The experiment has a number of rounds. Each round has two stages.

Stage 1:

- The Reds take turns to individually choose a Blue. The order in which the Reds get to choose a Blue is randomly determined.

- This results in 3 Red-Blue pairs with2 Blues left unmatched.

- The 2 Blues who have not been chosen in this round do nothing and their final earnings for the round are -25 points. [NEXT]

- Each Red chooses LEFT, MIDDLE or RIGHT.

- If a Red chooses LEFT: Red's earnings are -10 points, Blue's earnings are +15 points.

- If a Red chooses MIDDLE: Red's earnings are 0 points, Blue's earnings are 0 points.

- If a Red chooses RIGHT: Red's earnings are +15 points, Blue's earnings are -15 points. [NEXT]

Stage 2:

- Each Blue learns if they are in a pair with a Red.

- If they are in a pair, they will learn the action their Red partner chose among LEFT, MIDDLE and RIGHT, and the corresponding earnings in points.

- The paired Blues will choose 1 of 3 actions:

- To add 20 points to their Red partner at the cost of 5 points to them.

- To subtract 20 points from their Red partner at the cost of 5 points to them.

- Do nothing at zero cost. [NEXT]

- Red's final earnings will be changed by their Blue partner's choice to add or subtract. If Blue chose to subtract, then Red's earnings decrease by 20 points. If Blue chose to add, then Red's earnings increase by 20 points. In both cases Blue's earnings decrease by 5 points.

- If Blue does nothing, then Red's and Blue's final earnings are the points initially decided by the action chosen by the Red partner. 
- Blues who have not been chosen in this round do nothing and their final earnings for the round are -25 points. [NEXT]

That's a round. The experiment will last a number of rounds. [NEXT]

\section{Reputation treatment}

IDs:

- Reds and Blues will have IDs (e.g., Red 2 or Blue 4). Both Reds and Blues always keep the same ID.

- After every round, everyone will see the actions chosen by the 3 Red-Blue pairs up to and including that round. People's action choices are labeled by their ID.

We will begin the experiment now. Your role in the experiment will be decided in the next screen. After your role is decided, I will read the specific instructions for Reds and Blues. Please refrain from asking any questions until I finish reading these instructions. [NEXT]

Your ID for this round is Red ***. Your ID will be the same for all rounds. Specific instructions for Reds:

- The order in which you choose Blues as partners may change every round.

- If you are the first, you can pick any of the 5 Blues.

- If you are not the first, then you only pick from the Blues that were not chosen before.

- The 2 Blues that are not picked will earn -25 points for that round.

- Remember: both Reds' and Blues' IDs are always the same throughout the experiment.

- After choosing a Blue counterpart for the first stage, we ask that you choose LEFT, MIDDLE or RIGHT. The corresponding earnings are shown in the supplementary Figure.

- You will find out your Blue partner's choice. [OK]

Now the Blues. Your ID for this round is Blue ***. Your ID will be the same for all rounds. Specific instructions for Blues:

- If a Red picks you in a given round, you will find out which action your partner chose. The corresponding earnings are shown in the supplementary Figure.

- Then you will choose 1 action among 3 actions

- To add 20 points to your Red partner at the cost of 5 points.

- To subtract 20 points from your Red Partner at the cost of 5 points.

- Do nothing at zero cost.

- If a Red does not choose you, you will do nothing and your final earnings for this round are -25 points.

- Remember: both Reds' and Blues' IDs are always the same throughout the experiment. [OK]

\section{One-shot treatment}

IDs: 
- $\quad$ Reds and Blues will have IDs (e.g., Red 2 or Blue 4). Reds always keep the same ID. Blues get a random ID every round. (e.g. Blue 1 in round 1 may or may not be the same person as Blue 1 in round 2.)

- After every round, everyone will see the actions chosen by the 3 Red-Blue pairs up to and including that round. People's action choices are labeled by their ID.

We will begin the experiment now. Your role in the experiment will be decided in the next screen. After your role is decided, I will read the specific instructions for Reds and Blues. Please refrain from asking any questions until I finish reading these instructions. [NEXT]

Your ID for this round is Red ***. Your ID will be the same for all rounds. Specific instructions for Reds:

- $\quad$ The order in which you choose Blues as partners may change every round.

- If you are the first, you can pick any of the 5 Blues.

- If you are not the first, then you only pick from the Blues that were not chosen before.

- $\quad$ The 2 Blues that are not picked will earn -25 points for that round.

- Remember: Blues' IDs may change every round.

- After choosing a Blue counterpart for the first stage, we ask that you choose LEFT, MIDDLE or RIGHT. The corresponding earnings are shown in the supplementary Figure.

- Remember: you keep the same ID throughout the experiment.

- You will find out your Blue partner's choice. [OK]

Now the Blues. Your ID for this round is Blue ***. Your ID may change every round. Specific instructions for Blues:

- If a Red picks you in a given round, you will find out which action your partner chose. The corresponding earnings are shown in the supplementary Figure.

- Remember: your ID may change every round.

- Then you will choose 1 action among 3 actions

- To add 20 points to your Red partner at the cost of 5 points.

- To subtract 20 points from your Red Partner at the cost of 5 points.

- Do nothing at zero cost.

- If a Red does not choose you, you will do nothing and your final earnings for this round are 25 points.

- Reds always keep the same ID. [OK]

Check if there are any questions. If not, the game should start. If anyone asks about the number of rounds, the experimenter should simply repeat:

"The experiment will last a number of rounds."

The experiment should go on for 11 rounds or 70 minutes - whatever comes first. That leaves 20 minutes for paying people etc. 
Sample screenshot of a manager's choice:

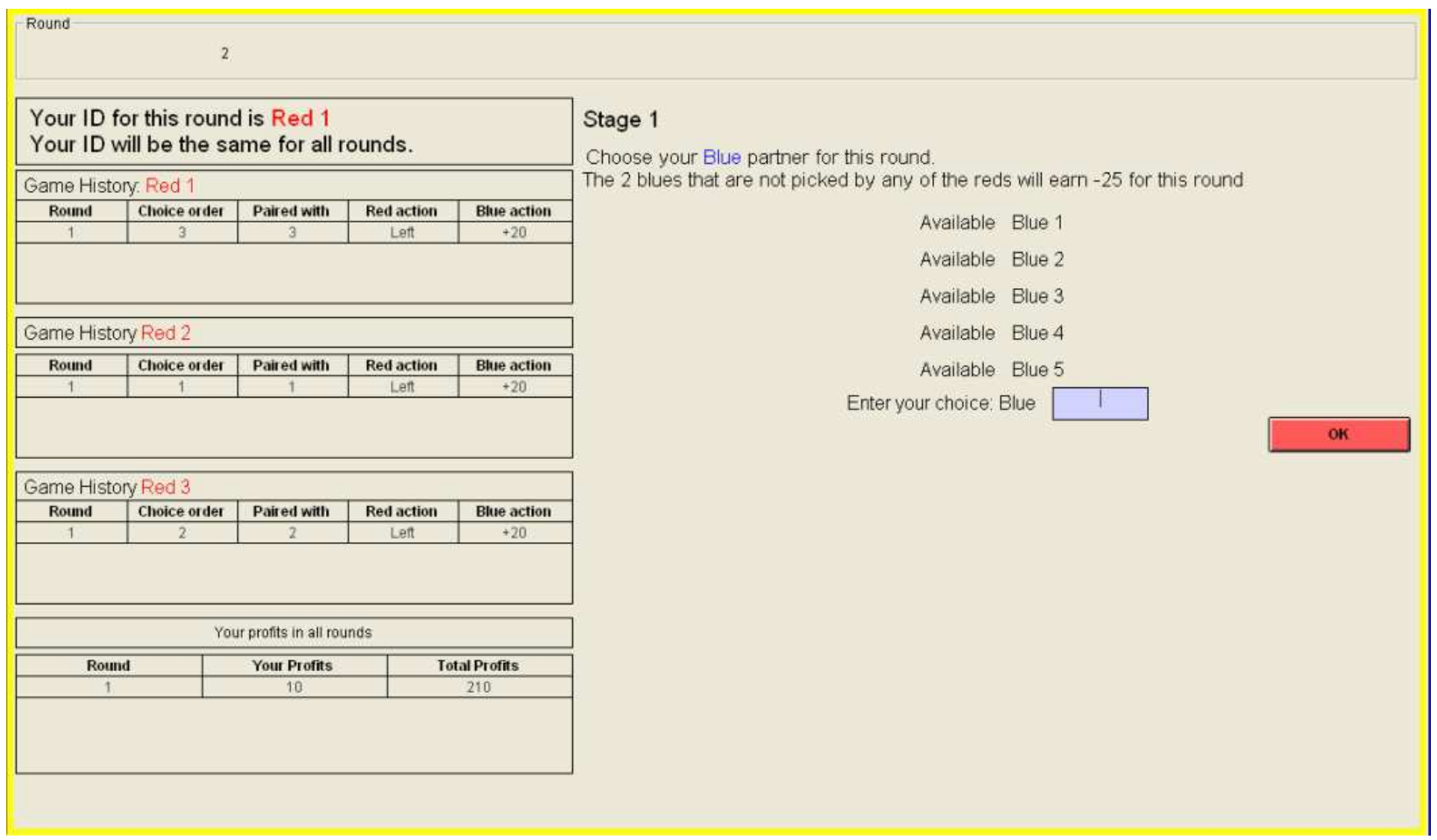

Sample screenshot of a worker's choice:

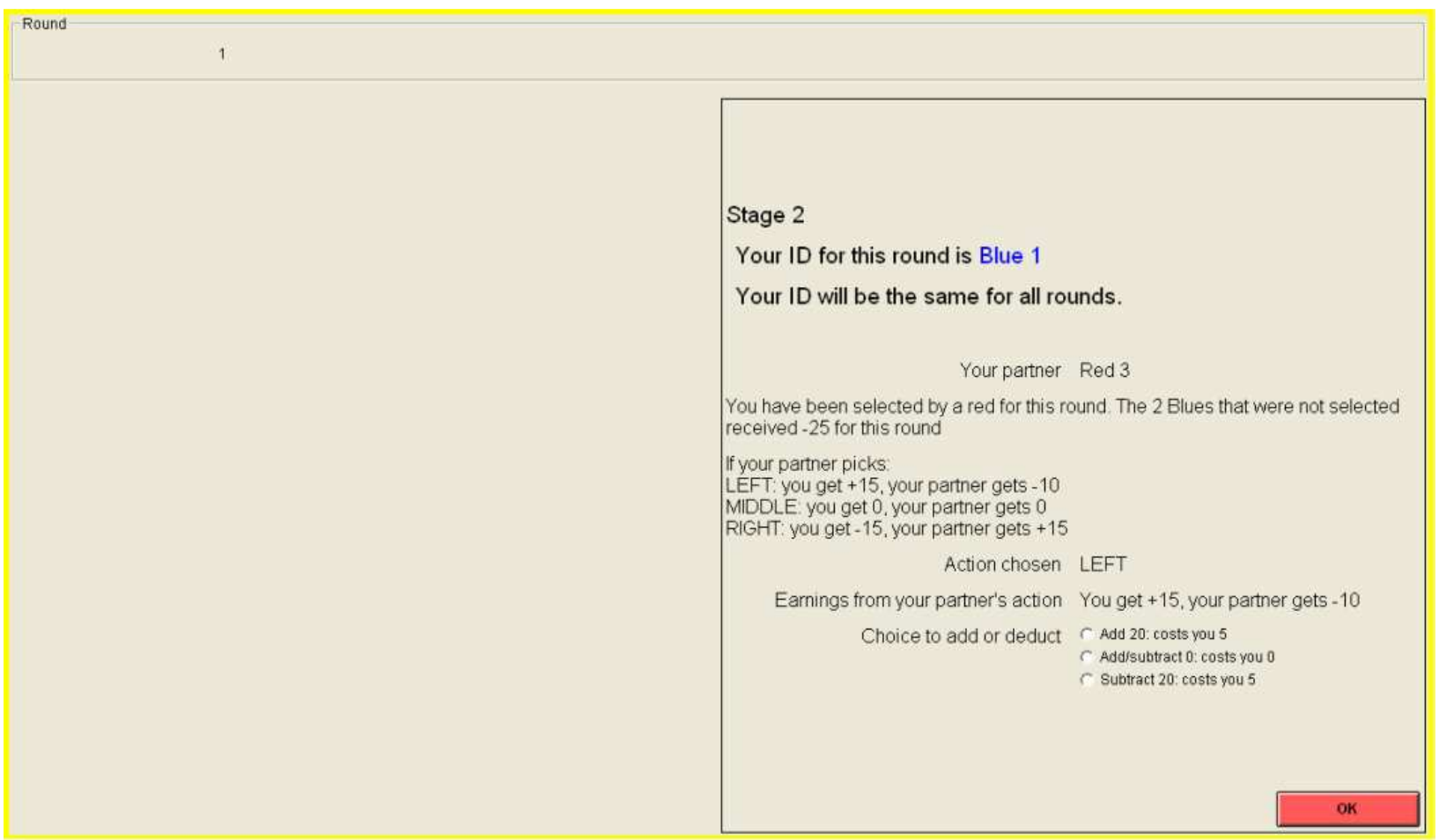

